\title{
The isodense colloid cyst: an easily overlooked cause of intermittent acute obstructive hydrocephalus
}

\author{
Mougnyan $\operatorname{Cox}^{1} \cdot$ Pranshu Sharma $^{1}$
}

Received: 18 September 2016/ Accepted: 22 September 2016/Published online: 5 October 2016

(c) SIMI 2016

\section{Case}

A 30-year-old woman presented to the emergency department with severe intermittent headaches over the course of 1 week. She denied any leg or arm weakness, and she reported no visual changes or loss of consciousness at any time. Due to the concern for intracranial pathology, a noncontrast CT scan of the head was performed. The CT scan showed dilatation of the lateral ventricles and diffuse sulcal effacement over both cerebral convexities (Fig. 1). Close inspection of the anterior third ventricle revealed a subtle isodense round mass consistent with an isodense colloid cyst causing acute obstructive hydrocephalus. Contrast-enhanced MRI of the brain was performed, and showed the colloid cyst to better advantage (Fig. 2). The patient underwent endoscopic resection of the cyst, and was symptom-free at follow-up with no evidence of recurrence.

Colloid cysts are usually hyperdense on CT scan (Fig. 3), making them readily visible and easy to diagnose [1]. These cysts are characteristically located in the anterior third ventricle, where they can cause intermittent obstruction and hydrocephalus, with neurological deterioration

Mougnyan Cox

mougnyan.cox@gmail.com

1 Thomas Jefferson University Hospitals, Philadelphia, PA, USA

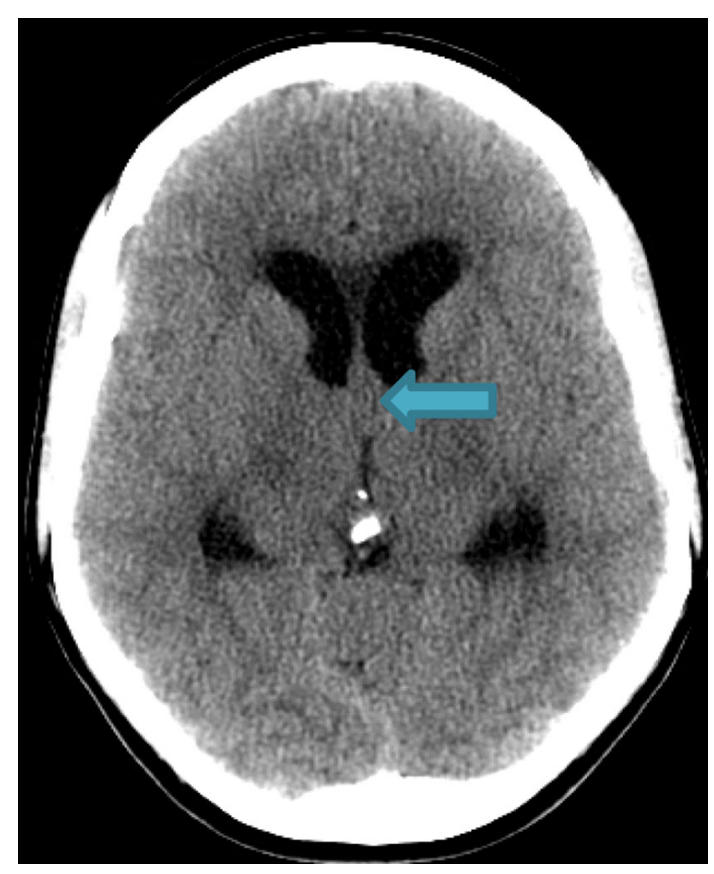

Fig. 1 Noncontrast head CT scan shows diffuse sulcal effacement and dilatated lateral ventricles. An isodense mass is present at the anterior third ventricle in the region of the foramen of Monro (arrow)

and death occurring in some patients [2]. Isodense colloid cysts may be easily overlooked, especially if unaccompanied by imaging findings of hydrocephalus (ventricular dilatation and sulcal effacement). In addition to sentinel intracranial hemorrhage related to aneurysm rupture, colloid cysts should be included in the diagnosis of young patients presenting with severe intermittent headaches, as this disorder is surgically curable [3]. 


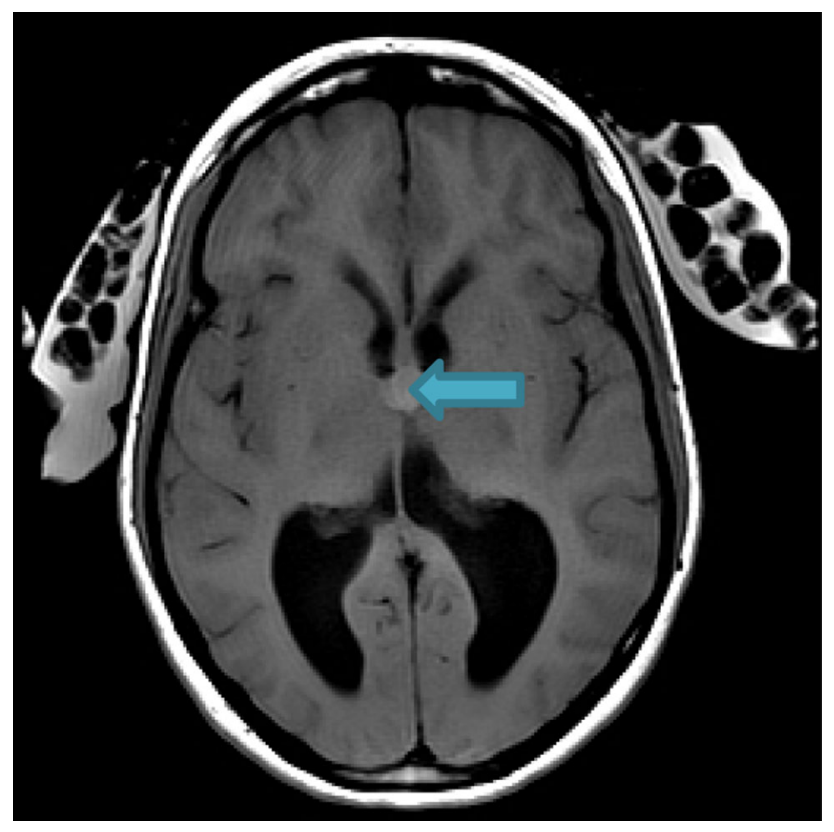

Fig. 2 T1-weighted MRI shows a round hyperintense mass in the region of the foramen of Monro (arrow). The intrinsic $T 1$ hyperintensity is related to proteinaceous products within the cyst. Ice packs were placed on the patient's forehead (at the request of the patient) during MRI imaging to help with her headache

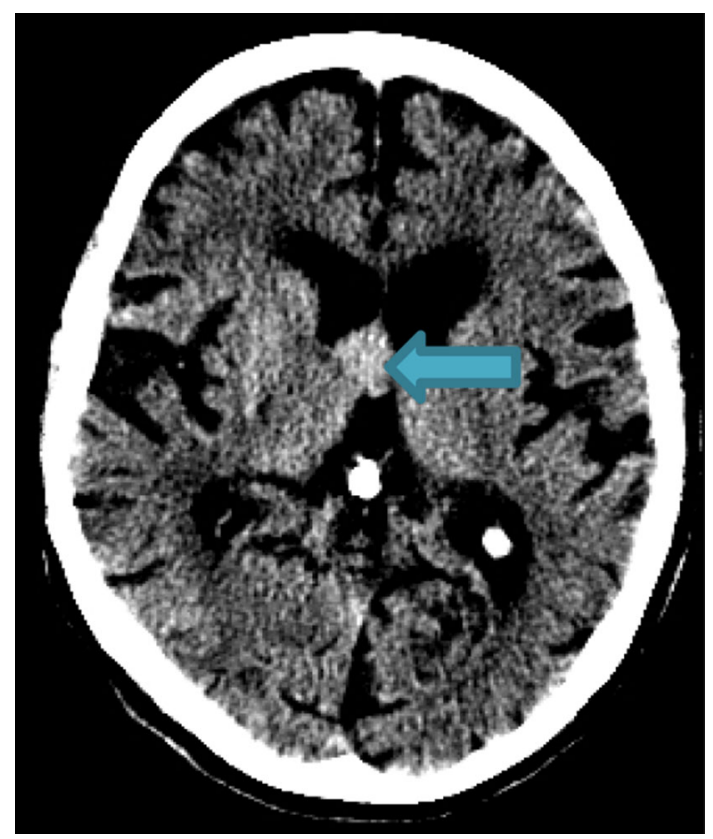

Fig. 3 Noncontrast CT in a different patient shows the classic appearance of a round hyperdense colloid cyst in the anterior third ventricle (arrow)

\section{Compliance with ethical standards}

Conflicts of interest None.

Statement of human and animal rights All studies involving Human subjects were compliant with ethical standards set forth by the institutional review board as well as the 1964 declaration of Helsinki and its later amendments. No animal studies were performed.

Informed consent Informed consent was obtained prior to all procedures in this study, and all personal identifiers were removed as required by the institutional review board.

\section{References}

1. Arma D, Castillo M, Chen $\mathrm{H}$ et al (2000) Colloid cyst of the third ventricle: imaging-pathologic correlation. Am J Neuroradiol 21: 1470-1477

2. Chan RC, Thompson GB (1983) Third ventricular cysts presenting with acute neurological deterioration. Surg Neurol 19:358-362

3. Horn E, Feiz-Erfan I, Bristol RE et al (2007) Treatment options for third ventricular colloid cysts: comparison of open microsurgical versus endoscopic resection. Neurosurgery 60:613-620 